\title{
Kinderurologische Jahreskonferenz: Plymouth, Mai 1991
}

\author{
R. Hohenfellner \\ Urologische Universitätsklinik Mainz
}

I. Aaronson (Charleston): Zur Untersuchung über mögliche Nebenwirkungen von submukös bzw. intravenös infiziertem Teflon bei Hunden.

Die „Sting-Therapie“ mit Teflon zur Behandlung des VUR wurde vor einigen Jahren eingeführt. Zwischen 1,4-2,5 ccm werden für die ein- oder beidseitige submuköse Antirefluxinjektion benötigt. Die genaue Zahl der weltweit damit behandelten Kinder und Jugendlichen kann nur grob geschätzt werden und dürfte bei über 2000 liegen.

Zur Kontrolle der Teflonnebenwirkungen bzw. -spätfolgen wurde Teflon in die Blasenwand von Hunden appliziert. Bereits 14 Tage später konnten Teflon-Partikel in der homogenisierten Lunge der getöteten Tiere nachgewiesen werden. Offenbar gelangt Teflon über den Blutstrom auch ins Gehirn, wo es bei 5000facher Vergrößerung nachweisbar war.

Bei weiteren elektronenmikroskopischen Untersuchungen an einer zweiten Versuchsserie fanden sich nach 16 Monaten Areale mit deutlicher Vaskulitis und Demyelinisierung rund um die Teflonpartikel in Pons und Medulla.

Die Diskussion unter den 24 Teilnehmern zeigte, daß nur zwei Teflon überhaupt verwenden und daß Versuche, dieses durch Eigenfettinjektion oder Knorpelzellenhomogenate zu ersetzen, fehlschlugen. Auch bei Verwendung von Kollagen können Nebenwirkungen nicht ausgeschlossen werden, abgesehen von berechtigten Zweifeln an der Dauerhaftigkeit der Refluxfreiheit. Bei höheren Konzentrationen wurden im Gewebe der Versuchstiere Kalzifikationen nachgewiesen.

Einem möglichen Zusammenhang zwischen Dickdarmkarzinom und Nierenanomalien ging J. Altwell (Southampton) nach. Untersucht wurden 58 Patienten aus 29 Familien mit 116 Großeltern. Erste Ergebnisse der noch nicht abgeschlossenen Studie: Wird bei Kindern mit einer Nierenanomalie, wie z.B. Doppelanlage und Ureterozele, eine genaue Familienanamnese erhoben und finden sich hierbei kolorektale Karzinome, so liegt die Wahrscheinlichkeit einer familiären Häufigkeit um das 118 fache höher.

M. Mitchell (Seattle): Bei 73 Patienten wurden gestielte Magensegmente vorwiegend aus der großen Kurvatur zur Blasenaugmentation und Substitution verwendet. Nur acht von diesen 72 zeigten eine Verschlechterung der Nierenfunktion. 63 sind tags und nachts kontinent,

Akt. Urol. 22 (1991) 395-396

(c) Georg Thieme Verlag Stuttgart · New York eingeschlossen Patienten unter CIC. Zu den wesentlichen Komplikationen zählt das Hämaturie-Dysurie-Syndrom in $22 \%$ aller Fälle. Patienten mit eingeschränkter Nierenfunktion sind von den schwer zu behandelnden mit perinealen und urethralen Schmerzen einhergehenden Hämaturien häufiger betroffen. H2-Rezeptorenblocker, Ditropan und Instillationen zur Phosphatneutralisation wurden mit wechselndem Erfolg verwendet. 9 Patienten hatten eine signifikante behandlungsbedürftige Alkalose mit erniedrigtem $\mathrm{Na}^{+}, \mathrm{K}^{+}, \mathrm{Cl}^{-}$und hohem $\mathrm{HCo}_{3}{ }^{-}$.

Unter den Diskussionsteilnehmern wurde die Operation im Vergleich zu den konventionellen Darmaugmentationen als technisch schwieriger gewertet. Eine sorgfältige Blutstillung bei der Magenresektion ist ebenso entscheidend wie ein spannungsloser "Gewebstransfer" ins kleine Becken. Ein mechanischer Ileus trat dennoch bei $10 \%$ der Patienten auf. Tierversuche zeigten ausschließlich benigne Polypen an der Magen-Blasen-Anastomose, jedoch keine Malignombildung. Bis auf weiteres dürfte die absolute Indikationsstellung (Fällen ohne verwendbare Darmsegmente wie kloakale Extrophie, herabgesetzte Nierenfunktion) überwiegen. Eine ausführliche Darstellung erscheint in einer der kommenden Ausgaben der Aktuellen Urologie (Operative Techniken).

Ein Ultraschallfarbdopplergerät wurde bei 31 Patienten mit akutem Skrotum (Durchschnittsalter 9,1 Jahre) für die Frage Hodentorsion?, Hydatidentorsion?, Epididymitis?, Orchitis?, Orchidoepididymitis?, Trauma? oder Tumor von $J$. Woodard (Atlanta) getestet. Zur Kontrolle diente der kontralaterale Hoden, bei 22 Patienten wurde zusätzlich eine Isotopenuntersuchung durchgeführt. In der Gruppe der Hydatidentorsionen war die Lernkurve am größten. Im oberen anterioren Segment fanden sich komplexe avaskuläre Bezirke im Ultraschall, eine Vergrößerung des Nebenhodens, eine reaktive Hydrozele und vermehrter Blutdurchfluß. Zusammenfassend konnte die Zahl der notwendigen Explorationen vermindert werden. Gemessene Parameter: longitudinaler und transvesikaler Durchmesser, Blutfluß im Hoden und Nebenhoden. Zum Beispiel fand sich bei der akuten Torsion ein vergrößerter Nebenhoden, kein Blutfluß im Hoden und ein reduzierter Blutfluß im Nebenhoden. In der Dikussion wurde betont, daß die Untersuchung der notfallmäßig eingewiesenen Patienten nur sinnvoll ist, wenn neben dem teuren Gerät auch der erfahrene Spezialist verfügbar ist. Allein der personelle Wechse] kann zu fatalen Fehlinterpretationen führen. Da der AFPWert bei Neugeborenen hoch ist, ist diese Untersuchung für die Differentialdiagnose Tumor wertlos.

Ein außerordentlich sorgfältig durchgeführtes pränatales Ultraschall-Screening von $D$. Thomas (Leeds) zum Nachweis des vesikorenalen Refluxes verlief enttäuschend. Im Gegensatz zur erwarteten hohen Inzidenz bei 
Mädchen war das Verhältnis männlich zu weiblich $6: 1$. Mit anderen Worten: infravesikale Obstruktionen (urethrale Klappen etc.) überwogen. Auch die zweite Fragestellung nach der weiteren Bedeutung einer sogenannten „milden postnatalen Dilatation“ erbrachte keinen Hinweis für die Ursache dieses häufig zu beobachtenden Phänomens und möglicher Spätfolgen.

Von 92 abnormalen Ultraschalluntersuchungen waren postnatal 47 normal. Im weiteren Followup fand sich nur zweimal ein VUR. Bei 20 Kindern mit einer minimalen Dilatation und einer Beobachtung von 3,8 Jahren war letztlich kein pathologischer Befund nachweisbar.

In der Diskussion wurde auf zahlreiche im pränatalen Schall übersehene Extrophien und Klappen hingewiesen - und erneut auf die qualitativen Unterschiede von Untersucher $\mathrm{zu}$ Untersucher. $\mathrm{Zu}$ wenig wird auf die Blasenwanddicke geachtet, die bei Knaben im Schnitt 2,6 mm beträgt. Ab 3,5 mm liegt ein sicher pathologischer Wert vor. Antenatal diagnostizierte Doppelsysteme wurden bei 32 Kindern postnatal von $C$. Schulman (Brüssel) weiterverfolgt. Das Verhältnis Knaben zu Mädchen betrug 5 : 1 . $30 \%$ der pathologischen Befunde normalisierten sich spontan, in 5 Fällen kam es zu einer Urosepsis. Eine konservative Therapie unter engmaschiger Kontrolle ist das Ziel weiterer Untersuchungen.

Nicht ohne Widerspruch blieb $J$. Scott's (Newcastle) Interpretation urodynamischer Befunde nach Blasenaugmentation bei Kindern vorwiegend mit detubularisertem Sigma. Das breite Spektrum postoperativ gefundener Hyperaktivität und spontaner Aktivität wurde vom Autor der Eigenbewegung des ausgeschalteten Darmsegmentes zugeordnet. Dagegen wurde eingewandt, daß urodynamische Untersuchungen mit gleichen Segmenten aber fehlendem Blasenrest (Blasensubstitution) zu anderen Ergebnissen führten. Die subtotale Detrusorresektion im Rahmen der Augmentation scheint demnach eine conditio sine qua non.

36 Patienten mit kindlichen Harnröhrenstrikturen überblickt E. Quesada (Buenos Aires). $60 \%$ waren traumatisch, $36 \%$ iatrogen bedingt und $4 \%$ kongenital ( $70 \%$ posteriore, $28 \%$ bulbäre Lokalisationen).

Die überwiegende Mehrzahl wurde nach primärer Verengung mit Zystostomie 3 bis 6 Monate später zur Rekonstruktion zugewiesen. Von den verschieden angewandten Techniken wie perineal, retropubisch und zweizeitig nach Bengt-Johannson erwies sich der kombinierte perineale retropubische Zugang mit End-zu-End-Anastomose als am erfolgreichsten. Restrikturierung (25\%) und Inkontinenz (14\%) wurde hauptsächlich nach bereits vorausgegangenen Rekonstruktionsversuchen beobachtet.

In den Händen von $S$. Arap (S. Paulo) erwies sich Asopas „transverse preputial island flap“ in 6 Fällen von langsreckigen hinteren Urethralstrikturen als erfolgreiche Methode zur tubulären Interposition mit End-zu-EndAnstomose oder zur Defektdeckung. Einziger bisheriger Nachteil war die nur radiologisch nachweisbare postoperative Dilatation der Neourethra bei fehlendem Corpus spongiosum.
H. Hendren (Boston) verwies auf ähnlich gute Ergebnisse mit freien Vollhauttransplantation und $G$. Monfort (Marseille) mittels Blasenmukosa. Eine für alle Verfahren entscheidende Rolle kommt der Lappendeckung bzw. der Unterpolsterung mit umliegendem Fettgewebe zu.

Wird während einer Urethroskopie eine „Öffnung am Boden der bulbären Urethra entdeckt“, so sollte man sich der möglicherweise erweiterten Ausführungsgänge der „Cowperschen Drüsen“ erinnern (P. Ransley, London). Meist asymptomatisch sind membranartige Zysten bei obliterierten Ausführungsgängen gelegentlich Ursache infravesikaler Obstruktion. A. Colodny (Boston) verwies auf die Differentialdiagnose der inkompletten Harnröhrenduplikation.

Als nach wie vor unbefriedigend müssen die Ergebnisse der Rekonstruktionsergebnisse der extrophen Blase bewertet werden (B. Churchill, B. Jeffs, B. O'Donnell). Der tags und nachts kontinente Patient mit einem normalen oberen Harntrakt ist eher die Ausnahme denn die Regel. Letzthin wird auch dieses ersehnte Ergebnis mit einer langen „inkontinenten Wartezeit“ (im Durchschnitt 8 Jahre), mehrfachen Operationen und nicht zuletzt mit Blasenaugementation und intermittierendem Katheterismus erkauft. Kritisch gesehen, ähnelt dieser immer häufiger gewählte Weg mehr einem Ausweg aus einer chirurgischen Sackgasse als einem Konzept zur Konstruktion eines kontinenten Reservoirs mit suffiziertem Auslaßwiderstand und willkürlicher Entleerung. Eine „Kontinenzzone“ von $40 \mathrm{~mm}$ Länge und ein Auslaßwiderstand von $60 \mathrm{~cm} \mathrm{H}_{2} \mathrm{O}$ werden empfohlen. Liegt jedoch der Blasendruck über $70 \mathrm{mH}_{2} \mathrm{O}$, finden sich in bis zu 95\% Störungen des oberen Harntraktes. Mit einer modifizierten Technik von Young-Dees erzielte $G$. Monfort (Marseille) die besten Ergebnisse bei der kontinenten Epispadie ( 7 von 9 Fällen) und die wenigsten Blasenentleerungsstörungen (4 von 21). Die sogenannte Autoaugmentation der Blase (partielle Detrusorresektion bei belassener Mukosa) führt nicht zur Erhöhung der Kapazität neurogener Blasen (S. Arap, S. Paulo, E. Quesada, Buenos Aires). Hingegen wird initial eine Verbesserung der Kontinenz beobachtet.

H. Hendren (Boston) und A. Retik (Boston) demonstrierten zum Abschluß Fälle mit kloakaler Extrophie, bei denen eine individuelle Rekonstruktionstechnik mit ausgeschalteten Darmanteilen und Magensegmenten angewendet wurde. Als eine allgemeine Empfehlung gilt der Verschluß der Omphalozele, die Vereinigung der zweigeteilten Blase, die Bildung eines Blasentubus zum späteren Einmalkatheterismus und die Defektdeckung mit einem gestielten Antrumsegment. Das infunktionelle blind in die Blasenplatte mündende mittlere Kolonsegment eignet sich kaum zum analen Durchzug, hingegen zum Vaginalersatz und manchmal auch als Urethraltubus. In drei Fällen wurde mit einem „ilealem" Nippel (perineales Stoma) und CIC eine Kontinenz der aufgebauten Blase erzielt.

Über schwerwiegende chirurgische Komplikationen bei der primären Wilms-Tumoroperation auch bei fortgeschrittenem Stadium wird in einem weiteren Beitrag von Kelalis in der Akt. Urologie berichtet.

\section{Prof. Dr. R. Hohenfellner}

Direktor der Urologischen Klinik und Poliklinik im Klinikum der Johannes-Gutenberg-Universität Langenbeckstr. 1

6500 Mainz 